\title{
Capacidade turística como pressuposto de desempenho organizacional: Um estudo no setor hoteleiro
}

\author{
Tourism capacity as organizational performance assumption: A study in the hotel sector
}

\author{
Elvis Silveira-Martins
}

Universidade Federal de Pelotas/Faculdade de Administração e de Turismo/Departamento de Administração - Campus Porto - Rua Gomes Carneiro, 1, 96.010-610 - Pelotas, Rio Grande do Sul, Brasil, elvis.professor@gmail.com

\section{Vinícius Costa da Silva Zonatto}

Universidade Regional de Blumenau/Programa de Pós-graduação em Ciências Contábeis/Departamento de Contabilidade - Rua Antônio da Veiga, 140, B. Victor Konder, 89.030-903 - Blumenau, Santa Catarina, Brasil, viniciuszonatto@gmail.com

\section{Luiz Eduardo Mascarenhas}

Universidade Federal de Pelotas/Faculdade de Administração e de Turismo/Departamento de Administração - Campus Porto - Rua Gomes Carneiro, 1, 96.010-610 - Pelotas, Rio Grande do Sul, Brasil, mascarenha9@gmail.com

\section{Resumo}

As capacidades turísticas procuram melhor aproveitar as competências internas, superando as adversidades ambientais e provendo melhores resultados para a empresa, apresentando-se como uma vertente das capacidades dinâmicas, com potenciais estratégicos para as tomadas de decisões. Desta maneira, objetiva-se com esta pesquisa relacionar a capacidade turística com o desempenho organizacional, em específico do setor hoteleiro. A pesquisa amparou-se em uma amostra de 65 hotéis, sendo que o tratamento dos dados deu-se por meio de análise fatorial confirmatória e modelagem de equações estruturais. Os resultados demonstraram que existe relação positiva e estatisticamente significante entre a capacidade turística e o desempenho dos hotéis pesquisados. Nestes casos, as capacidades turísticas influenciam positivamente o desempenho dos hotéis pesquisados. Conclui-se que a capacidade turística é adequada ao que preconiza a teoria seminal sobre capacidades dinâmicas, sendo fonte de atenção dos executivos para a melhoria da vantagem competitiva de suas organizações.

Palavras-chave: Capacidade turística, capacidades dinâmicas, desempenho, hotéis, vantagem competitiva.

\section{Abstract}

The tourism capabilities seek better use of internal skills, overcoming environmental adversities and providing better results for the company, presenting itself as a part of dynamic capabilities, with strategic potential for decision-making. Thus, in order to verify its effect on strategic outcomes, the purpose of this research is to relate the tourism capabilities with organizational performance, in particular in the hospitality industry. Research was based in a sample of 65 hotels, and the processing of data was given by confirmatory factor analysis and structural equation modeling. The results showed that there is a positive and statistically significant relationship between the tourism capabilities and performance of surveyed hotels. In these cases, the tourism capabilities positively influence the performance of surveyed hotels. It is concluded that the tourist capacity is adequate to what is advocated by the seminal theory of dynamic capabilities, a source of attention of executives to improve the competitive advantage of their organizations.

Keywords: Tourism capabilities, dynamic capabilities, performance, hotels, competitive advantage.

\section{Introdução}

A busca pela identificação da(s) fonte(s) de desempenho da organização instiga pesquisadores da área de estratégia no desenvolvimento de pesquisas sob os mais diversos ângulos teóricos e segmentos empresariais. Sob este prisma, verifica-se que as capacidades dinâmicas recebem atenção, pelo fato de que seu conceito seminal propõe que a mesma está associada com resultados superiores. Todavia, as capacidades quando apresentadas de maneira generalista parecem não representar a real finalidade a que se propõem, por conta da falta de particularidade e aderência ao escopo de pesquisa/ação.

Neste contexto, verifica-se que as capacidades turísticas se apresentam como fonte de análise direcionada especificamente para organizações do setor de turismo. Silveira-Martins e Zonatto (2016, p. 1) destacam que a capacidade dinâmica turística é compreendida como a "capacidade das organizações que atuam no setor turístico em gerenciar potencialidades internas da empresa com vistas a agregação de valor aos serviços prestados". Desta maneira, a capacidade turística apresenta-se como possível potencializadora de resultados organizacionais positivos em um segmento ímpar que, conforme é caracterizado por Phillips e Moutinho (2014), é intensivo e trespassa recursos naturais, culturais e sociais significativos.
Quando analisado o setor hoteleiro em particular, Marquesan, Guzzo, Zawislak e Tello-Gamarra (2015) destacam que este segmento vem recebendo maior atenção por pesquisadores da área de gestão e negócios, o que se deve a participação do setor de serviços, em especial o turismo, na geração de emprego e renda, o que impulsiona a economia do setor no país (Brasil). No entanto, pouco se sabe ainda sobre o desenvolvimento das capacidades organizacionais nestas empresas, bem como seu efeito em sua performance, lacuna teórica que estimula a realização de novos estudos sobre esta temática (Silveira-Martins \& Zonatto, 2016).

Desta maneira, tendo em vista a abordagem proposta por SilveiraMartins e Zonatto (2016), busca-se com este estudo correlacionar a capacidade turística com o desempenho organizacional, em específico de hotéis do município de São Paulo-SP (Brasil). Justifica-se o desenvolvimento desta pesquisa sob diferentes motes: i) recomendação de Silveira-Martins e Zonatto (2016) para o desenvolvimento de pesquisas que procurem analisar os efeitos da capacidade turística com o desempenho organizacional; ii) o conselho de Nieves e Haller (2014) de desenvolvimento de pesquisa avaliando o efeito das capacidades dinâmicas no desempenho das empresas hoteleiras; iii) não localização de estudos similares ao proposto, o que confere ineditismo à pesquisa; iv) o fato de proporcionar uma ferramenta com comprovação de resultados 
(desempenho) para os tomadores de decisões; e; v) ampliação dos estudos sobre capacidades dinâmicas.

O desenvolvimento da pesquisa será apresentado em cinco partes, afora esta introdução. Desta maneira na segunda seção serão apresentados os principais conhecimentos desenvolvidos sobre as temáticas capacidades turísticas e desempenho. Na sequência, serão apresentados os procedimentos metodológicos que nortearão a consecução do objetivo anteriormente enunciado. $\mathrm{Na}$ quarta seção serão apresentados e analisados os resultados dos tratamentos dos dados coletados. Em continuidade serão expostas as considerações finais do trabalho, seus limites de aplicação e proposições para agendas de pesquisas. Por fim, são apresentadas as referências utilizadas para o desenvolvimento da pesquisa.

\section{Marco teórico}

Nesta seção serão apresentadas as pesquisas basilares que darão suporte para o pleno desenvolvimento do estudo proposto, sem a pretensão de exaurir o debate e/ou apresentação sobre as temáticas. Para tanto, serão abordados os construtos capacidade turística e desempenho organizacional.

\subsection{Capacidade turística}

De acordo com Teece e Pisano (1994), a vantagem competitiva emerge a partir das capacidades dinâmicas das organizações. Nesta concepção, existe a congruência de duas perspectivas necessárias para o pleno desenvolvimento da estratégia por parte dos gestores, ou seja: capacidades e dinâmicas. Os autores enfatizam que as capacidades estão diretamente associadas a habilidade dos tomadores de decisões em adaptar, integrar e reconfigurar as competências internas e externas da organização, além dos recursos e competências funcionais em relação as mudanças ambientais. Em complemento a estas ações surge termo dinâmico, referindo-se as respostas estratégicas ao mercado, necessárias quando o tempo é exíguo e em contrapartida o ritmo de inovações é veloz, além da pouca disposição de informações sobre o futuro do mercado e da concorrência.

Neste contexto, Silveira-Martins e Zonatto (2016) propõem o conceito de capacidade turística como um mecanismo teórico/empírico que procura auxiliar os gestores e teóricos na interpretação da vantagem competitiva de organizações turísticas. Os autores conceituam a capacidade turística como sendo a gestão das potencialidades internas da empresa em prol do desenvolvimento das ações de turismo, antecedendo-se as ameaças ambientais e melhor administrando as oportunidades que Ihe são apresentadas, gerando assim, melhores resultados organizacionais. Este conceito vai ao encontro com os ensinamentos de Teece e Pisano (1994), quando destacam que para ser estratégica, a capacidade deve ser afinada a uma necessidade do usuário (para quem não são clientes), única (de modo que os produtos/serviços produzidos podem ser precificados sem dados relativos a concorrência), e difícil de replicar (de modo que os lucros não serão disputados fora da empresa).

Nesta perspectiva, Teece, Pisano e Shuen (1997) argumentam que a vantagem competitiva das organizações é derivada de orientações assumidas pelos gestores, coordenadas e combinadas, tais como: processos, posicionamento e caminhos estratégicos. Assim, os processos organizacionais são considerados como a forma de realização das atividades na empresa, suas rotinas, padrões de práticas atuais e aprendizagem. Já o posicionamento está associado com a adoção de tecnologias específicas, propriedades intelectuais, ativos complementares, base de clientes e suas relações externas com fornecedores e parceiros complementares. Por outra ótica, os caminhos estratégicos são entendidos como sendo as alternativas estratégicas disponíveis para as empresas, bem como a presença ou ausência de retornos crescentes, dependências de caminho e atendimento. Logo, se a vantagem competitiva é decorrida de processos, posicionamento e caminhos estratégicos, as capacidades dinâmicas devem possuir também estes fundamentos.

Deste modo, verifica-se na pesquisa de Silveira-Martins e Zonatto (2016) a presença coordenada e combinada das orientações identificadas por Teece, Pisano e Shuen (1997) como essenciais para a constituição de uma capacidade dinâmica, neste caso a capacidade turística proposta pelos autores. A constituição de um construto para avaliar uma capacidade específica de um determinado grupo de organizações, como a capacidade turística das empresas que atuam no setor do turismo, constitui-se uma nova oportunidade de pesquisa na área de gestão e negócios, o que reforça a necessidade de se explorar tais capacidades em empresas que atuam neste setor.

Na abordagem proposta por Silveira-Martins e Zonatto (2016), a variável geração de atendimento personalizado ao turista esta relacionada com processos, posicionamento e caminhos estratégicos. Por outra ótica, as variáveis: i) conhecimento sobre os atrativos turísticos (locais) do município e região; ii) em conjunto com atenção aos novos acontecimentos turísticos (locais) no município e região; e; iii) conhecimento sobre a história local e regional, representam as orientações processos e caminhos estratégicos. Já a variável localização próxima a pontos estratégicos associa-se diretamente com as orientações de posicionamento e caminhos estratégicos. Tais observações podem ser melhor identificadas no Quadro 1. 
Quadro 1 - Capacidades turísticas vs. orientação

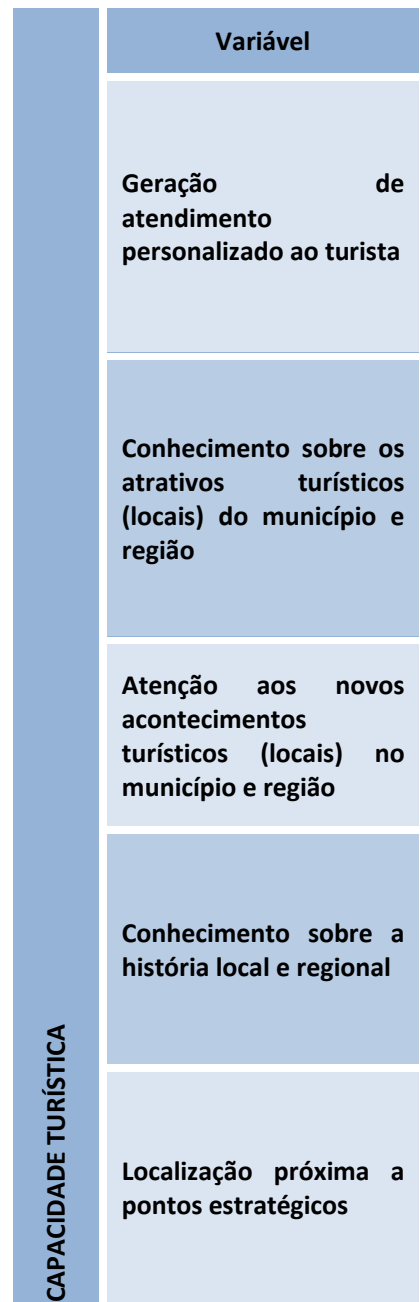

Descrição

Esta variável está associada com uma relação empática, prestativa e atenciosa com o turista, provendo atendimento individualizado e segmentado de acordo com suas necessidades. Incluem-se nesta variável, entre outros aspectos, o atendimento as necessidades específicas de idosos, condições de acesso para pessoas com deficiência física e/ou com mobilidade reduzida, integração entre hóspedes e animais de estimação, atendimento com fluência em diferentes idiomas falados e escritos.

A identificação de roteiros atrativos para as diferentes demandas de turistas está associada a esta variável. Para tal, os gestores devem estar atentos e prover uma variedade de opções presentes na localidade da organização e no seu entorno geográfico. Como por exemplo, pontos históricos, gastronômicos, lojas, belezas naturais (parques naturais, praias, montanhas), atrativos culturais (história/artes/museus), de diversão/entretenimento ( $p$. ex. festas populares, eventos esportivos), lazer em geral e centros comerciais/negócios.

Os aspectos que caracterizam esta variável estão intrinsecamente ligados a história recente da localidade e sua circunvizinhança. Entre os indicadores incluem-se o conhecimento sobre novas opções de lazer/entretenimento, como shows, exposições, eventos temáticos, esportivos, feiras de negócios.

A história local e regional perpetuada no decorrer dos anos sustenta esta variável. Desta forma, as informações turísticas devem contemplar fatos acontecidos, respeitando a simetria entre qualidade quantidade das mesmas, além de reconhecer o legado cultural da localidade/região. A título de exemplo incluem-se nesta variável, informações sobre colonização, personalidades, cultura, arte, tradições, costumes, economia.

Esta variável aborda aspectos relacionados com o posicionamento geográfico da organização e a infraestrutura turística. Neste sentido, devem ser apreciadas informações relativas a segurança dos turistas, transporte público/deslocamentos locais e regionais, proximidade com assistência médica, bares/restaurantes, aeroporto. Além destes aspectos outros relacionados a infraestrutura também estão associados, tais como: limpeza e preservação.
Orientação

Processos

Posicionamento

Caminhos Estratégicos

Processos

Caminhos Estratégicos

Processos

Caminhos Estratégicos

Processos

Caminhos Estratégicos

Posicionamento

Caminhos Estratégicos

Fonte: Adaptado de Silveira-Martins e Zonatto (2016).

É importante compreender adequadamente a distinção entre as capacidades, assim: i) conhecimento sobre os atrativos turísticos (locais) do município e região; ii) atenção aos novos acontecimentos turísticos (locais) no município e região; e, iii) conhecimento sobre a história local e regional, que podem parecer similares, mas referem-se a capacidades específicas. A primeira objetiva oferecer ao turista um mix ampliado de serviços que possam ser por ele utilizados. Já a segunda está relacionada à capacidade das empresas em reconhecer e proporcionar aos turistas, atrativos e potencialidades naturais e culturais de um dado lugar, o que se constitui novas alternativas, adicionais ao mix de serviços por ela desenvolvidos. Por sua vez, a terceira está relacionada ao conhecimento da carga histórica e cultural do(s) destino(s) turístico(s), a qual pode ser utilizada para estimular o interesse do turista em conhecer este local (Silveira-Martins \& Zonatto, 2016).

Eisenhardt e Martin (2000) posicionam-se reforçando o caráter dos processos no desenvolvimento das capacidades dinâmicas. Os pesquisadores destacam que as capacidades dinâmicas realmente consistem de rotinas específicas e identificáveis, que muitas vezes tem sido objeto de diversas pesquisas empíricas associadas diretamente a sua natureza, isto é, fora da teoria da visão baseada em recursos, sua antecessora. De toda a maneira, os autores, em conjunto com Kiessling e Harvey (2004), concordam que as capacidades dinâmicas firmam-se na competência de conquistar/manter a vantagem competitiva. Esta visão é compartilhada por Hung, Chung e Lien (2007), quando destacam que a capacidade dinâmica organizacional é uma mediadora para o processo de alinhamento organizacional com o desempenho.

Sob o prisma do turismo em especifico, Walsh, Lynch e Harrington (2011) defendem as capacidades dinâmicas, quando analisadas a luz da inovação, como fomentadoras de vantagem competitiva. Assim, de acordo com os autores, a inovação - caracterizada por um elevado grau de flexibilidade organizacional e implementação ativa e efetiva de novas estratégias e práticas organizacionais aumenta a produtividade e permite que empresas possam combinar sua base de recursos para os requisitos de um ambiente de negócios em rápida mudança. Isso dá peso ao argumento de que a inovação desempenha um papel inegável no sentido de ajudar pequenos empreendimentos de turismo a criar e sustentar uma vantagem competitiva.

Hu, Zhou, Daí, Zhan e Liu (2012) argumentam que a gestão da crise de empresas relacionadas ao turismo tem recebida muita atenção. No entanto, por se tratar de uma grande indústria formada por tipologias de negócios diferentes, por exemplo: hotéis, agências de viagens, entre outros, além de possuir uma relação estreita com o ambiente natural e social, imprime aos gestores desafios para a condução dos empreendimentos e resolver os problemas existentes. Assim, de acordo com estes autores, demandas de 
gestão de crises não se relacionam apenas com as normatizações organizacionais, mas também, com a comunicação rápida de informações, processamento de dados ágil e coordenação de todas as partes.

Neste sentido, a capacidade turística parece ser um mecanismo de gestão adequado a dar suporte aos tomadores de decisão deste segmento específico. Tal posicionamento ampara-se no ensinamento de Teece e Al-Aali (2013), quando os autores destacam que as capacidades dinâmicas ajudam a organização a desenvolver pontos fortes, estende-los, sincroniza-los com os modelos de negócios e com o ambiente de negócios e/ou, ainda, influencia o ambiente a seu favor.

Assim, sob a perspectiva do turismo e amparando-se em Vaz e Silveira-Martins (2016), pode-se empreender que a capacidade turística é formada por um conjunto de habilidades e características que fomentam uma habilidade singular as organizações de turismo, gerando diferenciação da forma como negociam com o ambiente. Tais elementos tendem a impactar positivamente no desempenho das organizações que atuam neste setor.

\subsection{Desempenho organizacional}

O desempenho organizacional tem sido abordado sobre diferentes aspectos e ângulos, tornando-se um construto multifacetado e abrangente. Neste sentido, observa-se que cientistas de diferentes linhas dos estudos estratégicos têm dedicado atenção a esta temática, procurando identificar qual a melhor rota a ser seguida pelo gestor para obter melhor desempenho e com isto posicionar suas organizações com vantagem competitiva.

Neste contexto, por exemplo, identifica-se o estudo de Haveman (1992), que aborda o desempenho sob a ótica da ecologia das organizações. De acordo com o autor, a inércia nas organizações não melhora as chances de sobrevivência quando as condições ambientais sofrem alterações. Assim, uma incerteza ambiental pode ser fator de extinção da organização. Haveman (1992) destaca que empresas que atuam em ambientes de mudanças intensas obrigam-se a ajustar suas estratégias e estruturas, a fim de não colocar em risco o desempenho organizacional.

Além do ambiente, outros fatores influenciam a atuação das empresas, bem como seu desempenho. Ergün e Tasgit (2013) verificaram a associação entre os tipos de cultura adhocrática (empreendedorismo), mercadológica (superioridade mercadológica), clã (tradição) e hierárquica (burocracia) e o desempenho inovador de empresas hoteleiras. Os resultados encontrados por estes autores evidenciaram que o desempenho é influenciado diretamente pelas características culturais. Ergün e Tasgit (2013) argumentam que a inovação é discutida como um elemento crítico para o sucesso de uma organização, mas se a cultura de uma organização não apoiar a inovação é improvável que ele ocorra. Os resultados desta pesquisa apontam ainda que o desempenho inovador dos hotéis tem características da cultura adhocrática e mercadológica, com resultados mais positivos do que a cultura clã e da hierárquica.

Por certo, empreendimentos hoteleiros localizados em economias em desenvolvimento devem criar estratégias competitivas eficazes para sua sobrevivência, uma vez que eles existem em um ambiente em que as partes interessadas estão exigindo mais de cada organização. Em consequência, devem concentrar-se em indicadores de desempenho fiáveis e críticos, absolutamente importantes para o sucesso de hotéis. Também devem adotar um quadro global de medição de desempenho que atenda aos requisitos do ambiente dinâmico em que está inserido (Kala \& Bagri, 2014). A pesquisa desenvolvida pelos cientistas apontou que os indicadores financeiros ainda são indicadores-chave de desempenho prevalentes e mais preferidos na gestão de quase todas as organizações de hospitalidade pesquisadas.

Corroborando este pensamento, verifica-se na pesquisa de Alberca-Oliver (2014) que ao analisar a eficiência setorial das empresas hoteleiras espanholas, valendo-se da perspectiva de dados microeconômicos e a relação com o resultado econômico das organizações, concluiu que as empresas mais eficientes são as pequenas e que obtiveram resultados econômicos positivos, sendo que a eficiência organizacional tende a diminuir proporcionalmente ao aumento do tamanho da empresa.

No entender de Afzal e Afzal (2014), as medidas que geram suporte ao desempenho de uma organização estão intimamente ligadas com a velocidade de desenvolvimento de capacidades baseadas em conhecimento que são geradas pela empresa. A este respeito, Ng e Tan (2015) destacam que o ambiente interno e externo devem ser fontes de análise para o desenvolvimento de estratégias com resultado na competitividade e desempenho organizacional. Tal abordagem é convergente a avaliação proposta nesta pesquisa, que investiga os efeitos da capacidade turística proposta por Silveira-Martins e Zonatto (2016) sobre a performance de empresas hoteleiras.

Nos últimos anos, várias iniciativas para a melhoria na consecução do desempenho superior e criação de valor para as organizações têm surgido (Castellanos, Ramos, Moreno \& González, 2016). Isso aumentou o interesse em investigar o processo de implementação de estratégias de melhoria e avaliação correspondente à luz dos objetivos e planos estratégicos corporativos. No entanto, não há ainda um consenso na literatura sobre qual a melhor forma de se avaliar o desempenho de uma empresa, e, por consequência tais efeitos sobre o mesmo.

No caso investigado nesta pesquisa, entende-se que um positivo desempenho organizacional pode ser alcançado com a formulação e implementação de estratégias que enfatizem as capacidades da organização, sendo possível, também, contornar as incertezas apresentadas pelo ambiente (Silveira-Martins \& Tavares, 2014a). Assim, a avaliação do desempenho das organizações hoteleiras pesquisadas se dará a partir de uma perspectiva econômica, em que serão observados indicadores de receitas, custos e margem de lucro. Tais indicadores tem sido utilizado (Carvalho, 2011; Castro Júnior, 2013) no país para a avaliação do desempenho de empresas hoteleiras.

\subsection{Hipótese de pesquisa}

Devido à escassez de estudos sobre a capacidade turística, por conta do ineditismo do construto, o que reforça a necessidade de desenvolvimento de pesquisas como essa, não foi possível 
identificar outras que procuraram analisar a relação entre a capacidade turística e do desempenho das organizações.

Por outro lado, Silveira-Martins e Zonatto (2016) destacam que a capacidade turística é uma ramificação, com aplicação específica, das capacidades dinâmicas. Sob esta ótica, é possível identificar a existência de outras pesquisas que se apoiaram em analisar a relação com o desempenho, a exemplo de: Hung, Chung e Lien (2007) - capacidade dinâmica organizacional (inovação e capacidade estratégica) e desempenho; Cabral, Coelho, Coelho e Costa (2015) - capacidade de exploração e explotação e desempenho; Silveira-Martins e Tavares (2014a) - capacidade mercadológica e desempenho; Silveira-Martins e Tavares (2014b) - capacidade gerencial e desempenho; Makkonen, Pohjola, Olkkonen e Koponen (2014) - detecção e aproveitamento, criação de conhecimento, integração, reconfiguração, alavancagem, aprendizagem e desempenho; Silveira-Martins, Rossetto e Añaña (2014) - capacidade ambidestra, exploração e explotação e desempenho; e Wilden, Gudergan, Nielsen e Lings (2013) - capacidade de detecção, aproveitamento de oportunidades, reconfiguração e desempenho, os quais encontraram evidências que sugerem uma associação positiva entre as capacidades dinâmicas e o desempenho organizacional (Silveira-Martins \& Vaz, 2016).

Aliado a este cenário, Krzakiewicz (2013) estimula o desenvolvimento de pesquisas com diferentes abordagens de capacidades dinâmicas, tendo em vista que as mesmas apresentam-se como um arcabouço teórico e empírico para o entendimento da vantagem competitiva das organizações.

Diante destas evidências, e considerando-se as proposições teóricas apresentadas por Silveira-Martins e Zonatto (2016) para o desenvolvimento de um construto específico para avaliar capacidades turísticas, elaborou-se a seguinte hipótese a ser avaliada neste estudo: $\boldsymbol{H}_{\mathrm{u}}$ : A capacidade turística correlaciona-se positivamente com o desempenho organizacional. A Figura 1 representa a $\mathrm{H}_{\mathrm{u}}$ em um modelo conceitual.

Figura 1 - Modelo conceitual

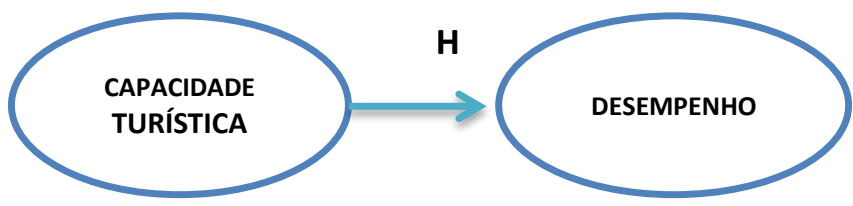

O método e os procedimentos utilizados para a realização desta pesquisa são apresentados a seguir.

\section{Procedimentos metodológicos}

Para a consecução da investigação, optou-se pela pesquisa quantitativa com o apoio da técnica survey. A amostra foi constituída de 65 hotéis, direcionados ao turismo, localizados em diferentes regiões do município de São Paulo-SP. Desta maneira, a amostra é classificada por Hair, Anderson, Tatham e Black (2009) como sendo por conveniência.

Os dados foram coletados com base em questionário fechado. Para o levantamento dos dados referentes ao construto capacidade turística, utilizou-se o instrumento desenvolvido e validado por Silveira-Martins e Zonatto (2016), sendo utilizada escala psicométrica de 1 a 7 , onde a menor nota deveria ser atribuída a pouca intensidade das ações e a maior nota a muita intensidade. Os indicadores foram classificados de CT1 a CT5, conforme Quadro 2.

\section{Quadro 2 - Variáveis construto capacidade turística}

\begin{tabular}{|l|l|}
\hline Construto & \multicolumn{1}{c|}{ Variável } \\
\hline & $\begin{array}{l}\text { CT1 - Geração de atendimento personalizado ao } \\
\text { turista }\end{array}$ \\
\hline $\begin{array}{l}\text { CT2 - Conhecimento sobre os atrativos turísticos } \\
\text { (locais) do município e região }\end{array}$ \\
\hline $\begin{array}{l}\text { CT3 - Atenção aos novos acontecimentos turísticos } \\
\text { (locais) no município e região }\end{array}$ \\
\hline$\frac{\pi}{0} \frac{0}{0}$ \\
\hline$\frac{0}{0}$
\end{tabular}

Fonte: Silveira-Martins e Zonatto (2016).

Para a coleta dos dados referentes ao desempenho organizacional, utilizou-se o instrumento desenvolvido e validado por Carvalho (2011), além de ter sido replicado por Castro Júnior (2013). Para o levantamento das informações realizou-se consulta junto aos gestores sobre alguns indicadores de desempenho, conforme Quadro 3, sendo solicitado a indicação, em uma escala psicométrica de 1 a 7 , de como percebia o desempenho do hotel frente a cada um dos indicadores, onde 1 representava baixo desempenho e 7 o inverso. As variáveis foram classificadas como Des1 a Des6.

\section{Quadro 3 - Variáveis construto desempenho}

\begin{tabular}{|c|c|}
\hline Construto & Variável \\
\hline \multirow{6}{*}{ 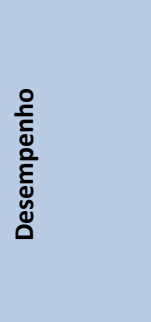 } & Des1 - Vendas totais \\
\hline & Des2 - Taxa média de ocupação \\
\hline & Des3 - Margem de lucro sobre as vendas totais \\
\hline & Des4 - Diária média \\
\hline & Des5 - Vendas por unidade habitacional \\
\hline & Des6 - Custo médio por diária vendida \\
\hline
\end{tabular}

Os dados foram coletados pelos pesquisadores por telefone, junto aos gestores administrativos dos hotéis selecionados para a pesquisa. A coleta de dados foi realizada no período compreendido entre julho e agosto de 2014.

Para o tratamento dos dados coletados foram utilizadas diferentes técnicas estatísticas. Inicialmente realizou-se o teste de normalidade de Kolmogorov-Smirnov com correção de Lilliefors, conforme recomendado por Hair et al. (2009) e Fávero, Belfiore, Chan e Silva (2009). Para esta atividade utilizou-se o software PASW Statistics 18.

Na sequência, realizou-se a análise fatorial confirmatória. Em função da ausência de normalidade dos dados, foi utilizado o estimador robusto de mínimos quadrados ponderados (WLSMV), conforme orientam Muthén e Muthén (2010). Neste sentido, verificou-se o fit dos indicadores ao modelo seguindo os parâmetros de qualidade recomendados por Hair et al. (2009). Assim, foram utilizados os indicadores: qui-quadrado (鞋), qui-quadrado dividido por graus de 
liberdade ([2]2/df) e o p-valor (p). Estes indicadores testam a hipótese nula de que a estimativa da covariância residual é igual a matriz nula, sendo que quando a hipótese nula é rejeitada, o modelo é considerado inadequado, por isso se almeja um valor de $\mathrm{l}^{2} / \mathrm{df}<3$ e o respectivo $p$-valor $>0,05$.

Além destes indicadores foram utilizados, também, o índice de ajuste comparativo (CFI), o índice de Tucker-Lewis (TLI) e o root meand square error of aproximation (RMSEA). Estes índices, bem como os valores almejados, podem ser observados no Quadro 4. Para o desenvolvimento dos cálculos foi utilizado o software MPlus, versão 7.

\begin{tabular}{|l|c|}
\multicolumn{2}{|c|}{$\begin{array}{c}\text { Quadro 4 - Testes de validade do construto } \\
\text { Indicador }\end{array}$} \\
\hline \begin{tabular}{|l|c|} 
Valores almejados \\
]$^{2}$
\end{tabular} \\
\hline$]^{2} / \mathrm{df}$ & $<3$ \\
\hline $\mathrm{p}$ & $>0,05$ \\
\hline $\mathrm{CFI}$ & $>0,900$ \\
\hline TLI & $>0,900$ \\
\hline RMSEA & $<0,10$ \\
\hline \multicolumn{2}{|c|}{ Fonte: Adaptado de Hair et al. (2009). } \\
\hline
\end{tabular}

Com base nos resultados aferidos na análise fatorial exploratória, realizou-se a modelagem de equações estruturais. Este procedimento foi realizado com base no software MPlus, versão 7. A estrutura proposta para o modelo inicial pode ser observada na Figura 2.

Figura 2 - Modelo inicial

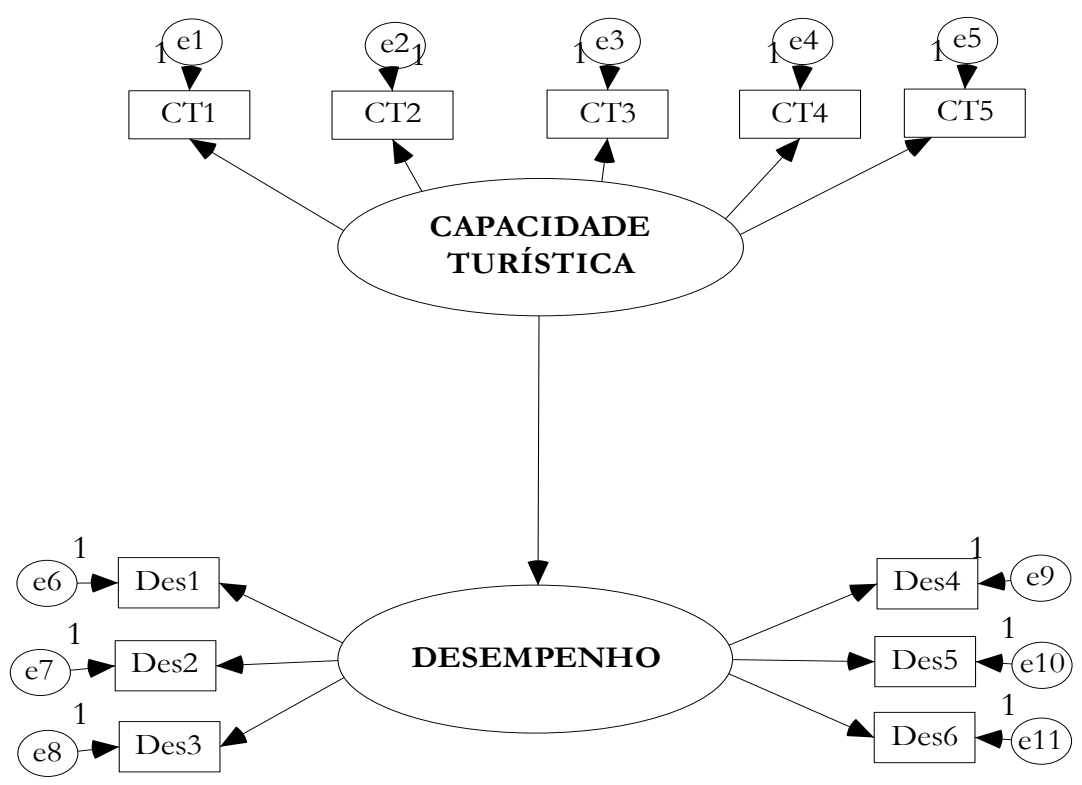

Assim, com base nos procedimentos anteriormente apresentados, na sequência serão apresentados os descritivos resultantes do tratamento dos dados, bem como a análise dos resultados encontrados no estudo.

\section{Análise dos dados}

A primeira análise dos dados foi direcionada ao teste de normalidade dos mesmos. Para tanto, por conta do tamanho da amostra e seguindo as recomendações de Fávero et al. (2009), realizou-se o teste de Kolmogorov-Sminorv com correção de Lilliefors. O teste resultou em uma estatística variando entre 0,134 a 0,342 entre as variáveis, sendo que o resultado de $p$-valor aferido para todas as variáveis foi menor do que 0,05 , logo, rejeita-se a hipótese nula. Este resultado aponta que a distribuição dos dados não apresenta normalidade. Todos estes apontamentos podem ser observados no Quadro 5.

Quadro 5 - Teste de normalidade

\begin{tabular}{|c|c|c|c|}
\hline \multirow{2}{*}{ VARIÁVEL } & \multicolumn{3}{|c|}{ KOLMOGOROV-SMIRNOV } \\
& Estatística & Graus de Liberdade & Significância \\
\hline CT1 & 0,230 & 64 & 0,000 \\
\hline CT2 & 0,178 & 64 & 0,000 \\
\hline CT3 & 0,186 & 64 & 0,000 \\
\hline CT4 & 0,134 & 64 & 0,006 \\
\hline CT5 & 0,236 & 64 & 0,000 \\
\hline DES1 & 0,232 & 64 & 0,000 \\
\hline DES2 & 0,205 & 64 & 0,000 \\
\hline DES3 & 0,240 & 64 & 0,000 \\
\hline DES4 & 0,342 & 64 & 0,000 \\
\hline DES5 & 0,259 & 64 & 0,000 \\
\hline DES6 & 0,296 & 64 & 0,000 \\
\hline
\end{tabular}


Na sequência realizou-se a análise fatorial confirmatória (AFC) do construto capacidade turística. Tendo em vista os resultados encontrados no Quadro 5, optou-se pela utilização do estimador WLSMV que, de acordo com Muthén e Muthén (2010), utiliza a matriz com pesos diagonais com erros padrão e médio - e variância - ajustado ao teste estatístico do qui-quadrado que utilizam uma matriz de peso.

Assim, após a realização do modelo (inicial), verificou-se que o [? ${ }^{2}$ apresentou o resultado de $17,854(\mathrm{df}=5)$, sendo que o resultado [? ${ }^{2} / \mathrm{df}$, apesar de próximo, não atendeu ao valor máximo almejado $(3,570>3)$. Neste contexto, observou-se o valor do $p$-valor $(0,003$ $<0,05)$. Por outra ótica os valores de CFI $(0,970>0,900)$, TLI $(0,939$ $>0,900)$ e $\operatorname{RMSEA}(0,008<0,10)$ atenderam ao que preconiza a literatura.

Tendo em vista que alguns indicadores não apresentaram o fit aconselhado, procurou-se desenvolver um modelo opcional. Conforme Hair et al. (2009) este procedimento é chamado de reespecificação do modelo, que pode ser utilizado para corrigir parâmetros inadequados encontrados no processo de estimação ou para criar um modelo concorrente de comparação. Todavia os autores alertam que este tipo de ação deve ser tratado com parcimônia quando existe exclusão de alguma variável.

Com base nos apontamento de Hair et al. (2009), para este segundo modelo, considerou-se apenas variâncias compartilhadas, sendo estas: CT1 com CT3 e CT1 com CT5. Este procedimento foi apontado pelo software MPlus, como alternativa para a melhoria dos indicadores de ajuste.

Na sequência, verificou-se que o valor do $]^{2}$ passou para 7,455 $(\mathrm{df}=3)$, resultando em $\mathrm{Q}^{2} / \mathrm{df}$ de $2,485(<3)$. O p-valor passou para $0,058(<0,05)$. Já os valores de CFI $(0,989)$, TLI $(0,965)$ e RMSEA $(0,092)$ permaneceram no intervalo identificado pela literatura como possuindo ajuste. Diante destes resultados, optou-se pela utilização do modelo opcional. 0 Quadro 6 apresenta, comparativamente, os resultados encontrados nos dois modelos.

\section{Quadro 6 - Indices da AFC Capacidade Turística}

\begin{tabular}{|c|c|c|c|c|}
\hline Estimador & Indicador & $\begin{array}{l}\text { Modelo } \\
\text { Inicial }\end{array}$ & $\begin{array}{l}\text { Modelo } \\
\text { Opcional }\end{array}$ & $\begin{array}{c}\text { Valores } \\
\text { Almejados }\end{array}$ \\
\hline \multirow{6}{*}{$\sum_{3}^{\gtrless}$} & ]$^{2}$ & $17,854(d f=5)$ & $7,455(\mathrm{DF}=3)$ & \\
\hline & ? $]^{2} / \mathrm{df}$ & 3,570 & 2,485 & $<3$ \\
\hline & $P$ & 0,003 & 0,058 & $>0,05$ \\
\hline & CFI & 0,970 & 0,989 & $>0,900$ \\
\hline & TLI & 0,939 & 0,965 & $>0,900$ \\
\hline & RMSEA & 0,008 & 0,092 & $<0,10$ \\
\hline
\end{tabular}

Prosseguindo a análise dos dados, procedeu-se a AFC do construto desempenho. Observou-se que o indicador ? $^{2}$ resultou no valor de 17,636 ( $d f=9$ ), sendo que ? $^{2} / \mathrm{df}$ apresentou o montante de 1,959 (< $3)$, sendo adequado aos parâmetros estipulados. Por outro prisma, o p-valor ficou abaixo do mínimo almejado $(0,039<0,05)$. Já os valores de CFI $(0,952>0,900)$, TLI $(0,921>0,900)$ e RMSEA $(0,084<0,10)$ apresentaram-se de acordo com o que preconiza a literatura.

Tendo em vista a busca pelo melhor fit do modelo, procurou-se desenvolver um modelo alternativo para efeito de comparação de resultados. Desta maneira, para o segundo modelo (opcional), foi considerado que as seguintes variáveis possuem variância compartilhada: Des1 com Des4; Des4 com Des2; Des1 com Des6; Des4 com Des3 e Des6 com Des3.

Após este procedimento, verificou-se que o valor de $?^{2}$ alterou para 8,895 ( $\mathrm{df}=4)$, por conseguinte $\mathbf{l}^{2} / \mathrm{df}$ passou para 2,223, permanecendo abaixo de 3 . Em relação ao $p$-valor, este aumentou para 0,063, estando acima do mínimo esperado, ou seja, 0,05, diferentemente do modelo inicial. Da mesma maneira, os valores de CFI $(0,973>0,900)$, TLI $(0,901>0,900)$ e RMSEA $(0,973<0,10)$, sofreram alteração. No entanto, permaneceram em conformidade com os parâmetros de ajuste. Tendo em vista o melhor fit proporcionado pelo modelo opcional, decidiu-se pela utilização deste para a realização dos demais procedimentos de análise. Os resultados encontrados podem ser observados no Quadro 7.

\begin{tabular}{|c|c|c|c|c|}
\hline Estimador & Indicador & $\begin{array}{l}\text { Modelo } \\
\text { Inicial }\end{array}$ & $\begin{array}{l}\text { Modelo } \\
\text { Opcional }\end{array}$ & $\begin{array}{c}\text { Valores } \\
\text { Almejados }\end{array}$ \\
\hline \multirow{6}{*}{$\sum_{\substack{3 \\
3}}^{\longrightarrow}$} & 国 ${ }^{2}$ & $17,636(d f=9)$ & $8,895(d f=4)$ & \\
\hline & 国 ${ }^{2} / \mathrm{df}$ & 1,959 & 2,223 & $<3$ \\
\hline & $\mathrm{P}$ & 0,039 & 0,063 & $>0,05$ \\
\hline & CFI & 0,952 & 0,973 & $>0,900$ \\
\hline & TLI & 0,921 & 0,901 & $>0,900$ \\
\hline & RMSEA & 0,084 & 0,100 & $<0,10$ \\
\hline
\end{tabular}

Valendo-se dos modelos individualizados de cada construto (capacidade turística e desempenho), após a execução da análise fatorial confirmatória, realizou-se os cálculos dos indicadores fit do modelo de equações estruturais relacionando a capacidade turística com o desempenho.
Desta maneira, após os procedimentos estatísticos, verificou-se que o indicador ? $^{2}$ apresentou o valor de $74,313(\mathrm{df}=36)$, sendo que $]^{2} / \mathrm{df}$ resultou em $2,064(<3)$. O valor do $p$-value resultou em 0,000 . Já os valores de CFI $(0,929>0,900)$ e RMSEA $(0,002<0,10)$ apresentaram-se nos parâmetros especificados. $\mathrm{O}$ indicador TLI 
$(0,892)$ infelizmente não é superior a 0,900, no entanto resultou em um valor muito próximo ao esperado. Diante deste cenário, além de uma análise contextual, entendeu-se que os indicadores demonstram que o modelo possui o fit necessário para a continuidade da análise proposta neste estudo. Assim, o modelo inicial será considerado na sequência do trabalho. Os resultados identificados podem ser observados no Quadro 8.

Quadro 8 - Indices da AFC Modelo

\begin{tabular}{|c|c|c|c|c|}
\hline Estimador & Indicador & $\begin{array}{l}\text { Modelo } \\
\text { Inicial }\end{array}$ & $\begin{array}{l}\text { Modelo } \\
\text { Opcional }\end{array}$ & $\begin{array}{c}\text { Valores } \\
\text { Almejados }\end{array}$ \\
\hline \multirow{6}{*}{$\sum_{n}^{\longrightarrow}$} & $\theta^{2}$ & $74,313(d f=36)$ & - & \\
\hline & ]$^{2} / \mathrm{df}$ & 2,064 & - & $<3$ \\
\hline & $\mathrm{P}$ & 0,000 & - & $>0,05$ \\
\hline & CFI & 0,929 & - & $>0,900$ \\
\hline & TLI & 0,892 & - & $>0,900$ \\
\hline & RMSEA & 0,002 & - & $<0,10$ \\
\hline
\end{tabular}

Com base nos apontamentos descritos, passou-se à análise dos coeficientes padronizados dos indicadores, como se pode observar na Figura 3. Pode se verificar que o menor coeficiente é 0,532
(Des4). Hair et al. (2009) orientam que a carga mínima a ser aceita é de 0,500 para ser confirmada a validade convergente, o que é percebido no modelo apresentado.

Figura 3 - Modelo final

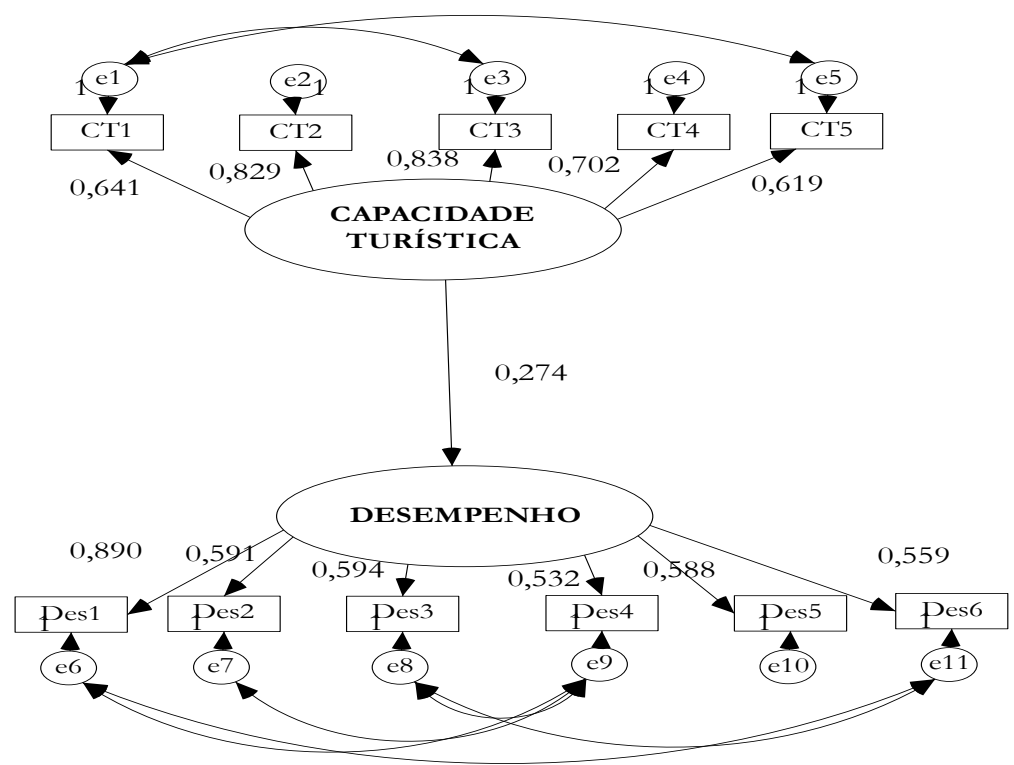

Baseando-se no modelo geral de mensuração das relações avaliadas nesta pesquisa (Figura 3), verificou-se uma relação positiva entre a capacidade turística e o desempenho das organizações pesquisadas $(0,274)$, sendo esta estatisticamente significativa a $0,05(p=0,000<0,05)$. Desta forma, a hipótese única elaborada para esta pesquisa pode ser confirmada.

Os resultados encontrados revelam que as capacidades turísticas dos hotéis pesquisados estão relacionadas a atenção aos novos acontecimentos turísticos locais e ao conhecimento sobre os atrativos locais. Conforme explicam Silveira-Martins e Zonatto (2016), conhecer a história da localidade e região, bem como suas opções de lazer e entretenimento constituem-se um diferencial de empresas que atuam no setor turístico para atender as diferentes necessidades de seus clientes.

A identificação de opções diferenciadas de entretenimento e lazer contribui para com a capacidade da organização de atender a diferentes públicos, cuja expectativa pode variar de indivíduo para indivíduo. Da mesma forma, a identificação de roteiros atrativos e variados contribui para que o turista encontre diferentes opções para consumo. Tais elementos impactam positivamente no seu desempenho. Portanto, estes resultados sugerem que o desenvolvimento das capacidades turísticas pelas empresas hoteleiras, como visto neste caso, impulsionam o seu desempenho.

Estes resultados possuem importantes implicações teóricas e empíricas. Sob a perspectiva teórica, estes achados sugerem a confirmação da teoria das capacidades turísticas (CT), conforme destacado por Silveira-Martins e Zonatto (2016), como sendo uma vertente das capacidades dinâmicas, tal como preconizado por Teece e Pisano (1994) e Teece e Al-Aali (2013), quando afirmam que as capacidades dinâmicas são competências/capacidades que possibilitam as organizações alcançarem melhores resultados, que, segundo Einsenhardt e Martin (2000), não podem ser definidas genericamente. 
Sob a perspectiva empírica, a CT apresenta-se como um instrumento de atenção, e consideração, para os gestores no momento de suas formulações de estratégias, tendo em vista os resultados aqui aferidos, ou seja, quanto maior o investimento sobre a CT maior tende a ser o retorno que a empresa obterá em termos de desempenho operacional e financeiro.

Quando confrontados estes resultados com o preconizado por Silveira-Martins e Tavares (2014a), verifica-se que o tomador de decisões que ampara-se na formulação de suas estratégias enfatizando as capacidades turísticas habilita-se a angariar resultados e, por conseguinte, vantagem competitiva sobre os players presentes no ambiente (Kiessling \& Harvey, 2004; Walsh, Lynch \& Harrington, 2011). Desta maneira, estes resultados contribuem para amenizar as angústias de um setor específico, conforme explanam Hu et al. (2012), apresentando uma rota estratégica que poderá servir de auxílio aos tomadores de decisões no cotidiano gerencial.

Em termos de desempenho, os resultados desta pesquisa apresentam-se em concordância com os achados de Wang e Ahmed (2007), Ambrosini e Bowman (2009), Tondolo e Bitencourt (2014) e Vaz e Silveira-Martins (2016), sendo considerado, este construto, proveniente das capacidades dinâmicas. Todavia, Ambrosini e Bowman (2009) destacam que Helfat et al. (2007) acreditam que as capacidades dinâmicas não geram obrigatoriamente vantagem competitiva, uma vez que, enquanto as capacidades dinâmicas podem alterar a base de recursos, esta renovação pode não ser necessariamente valiosa, ela pode não criar quaisquer recursos com valor, raridade, dificuldade de imitar ou substituir (VRIS), fundamento da visão baseada em recursos e precursora das capacidades dinâmicas. Sendo assim, as evidências encontradas nesta pesquisa estimulam a realização de novos estudos, uma vez que exploram uma capacidade específica de um determinado grupo de empresas, o que abre novas perspectivas teóricas de investigação para o tema.

\section{Considerações finais}

Objetivou-se com esta pesquisa correlacionar a capacidade turística com o desempenho organizacional em específico de hotéis do município de São Paulo-SP/Brasil. Procurou-se verificar a validade do construto capacidade turística, conforme sua concepção, em relação aos reais efeitos produzidos sobre o desempenho das organizações. Após a análise dos dados, pôde-se concluir que as capacidades turísticas possuem implicações diretas e positivas sobre os resultados dos hotéis. Este apontamento é congruente com a teoria sobre as capacidades dinâmicas, vistas como precursoras de desempenho.

Os resultados encontrados sugerem que investimentos em estratégias que abarquem ações efetivas que priorizem a geração de atendimento personalizado ao turista (cliente), geração e atualização permanente de conhecimento sobre os atrativos existentes, além da incorporação dos novos, e a proficiência sobre a história local e regional contribuem para o desenvolvimento de capacidades específicas para as empresas que atuam no setor turístico, melhorando o seu desempenho. Não se pode desconsiderar o posicionamento do hotel em termos de localização, uma vez que a proximidade, aliada aos demais indicadores, consolidam-se como fonte de desempenho das organizações hoteleiras, por sua vez, um posicionamento estratégico a ser considerado pelos gestores no momento da tomada de decisões.

Estes achados reduzem a fronteira ente o teórico (ciência) e o empírico (tomador de decisões), apresentando, em conformidade com a demanda de Nieves e Haller (2014), resultados práticos sobre influência das capacidades dinâmicas, abordando uma tipologia singular, e contrapondo-a com os resultados organizacionais. Tais evidências estimulam a realização de novos estudos, apresentando uma nova perspectiva de investigação para a temática.

Torna-se importante ressaltar que a pesquisa limita-se na amostra investigada, o que não é fonte de demérito do trabalho, mas de ponderação dos resultados que não podem ser generalizados e/ou extrapolados para um contexto diferente daquele em que foram realizadas as simulações. Assim, diante tais evidências, como proposta para as agendas de pesquisa de estudiosos da temática, sugere-se a ampliação desta pesquisa em outras regiões geográficas, considerando-se também a replicação em outros países, visando verificar se os resultados aqui aferidos se confirmam.

Recomenda-se a replicação deste estudo, também, em outros segmentos que se valem do turismo diretamente como enoturismo, zitoturismo, restaurantes, e indiretamente como concessionárias de táxi, empresas de câmbio, entre outros. A inserção do construto ambiente como mediadora da relação entre capacidade turística e desempenho também é fonte de recomendação para futuras pesquisas. Em função do apontamento de Tachizawa, Pozo e Vicente (2013) e Limberg, Boaria e Anjos (2014) de que o setor hoteleiro representa um dos segmentos que necessita atenção em relação às tecnologias da informação e de que é consideravelmente exposto às mídias sociais, recomenda-se a incorporação de uma variável que considere estes apontamentos em novas investigações, analisando-se seu comportamento no modelo.

\section{Referências}

Afzal, M., \& Afzal, U. (2014). Effect of knowledge management practices (KMPs) and the moderating role of interpersonal trust (IPT) on firm's performance (FP): a study in software industry of Pakistan. African Journal of Business Management, 8(19), 864-872.

Alberca-Oliver, P. (2014). ¿lncide el resultado contable y la dimensión empresarial en la eficiencia de las empresas hoteleras? Revista de Turismo y Patrimonio Cultural, 12(2), 299-314.

Ambrosini, V., \& Bowman, C. (2009). What are dynamic capabilities and are they a useful construct in strategic management? International Journal of Management Reviews, 11(1), 29-49.

Cabral, J. E. O., Coelho, A. F. M., Coelho, F. J. F., \& Costa, M. P. B. (2015). Capabilities, innovation, and overall performance in Brazilian export firms. Revista de Administração Mackenzie, 16(3), 76-108.

Carvalho, C. E. (2011). Relacionamento entre ambiente organizacional, capacidades, orientação estratégica e desempenho: um estudo no setor hoteleiro brasileiro. Tese de Doutorado, Programa de Pós-Graduação em Administração, Universidade do Vale do Itajaí, Biguaçu, SC, Brasil..

Castellanos, E. E. D., Ramos, C. D., Moreno, L. A. B., \& González, B. P. (2016) Estudio empírico para la validación de un modelo matemático que mide e desempeño corporativo em industrias manufactureras. Revista Global de Negocios, 4(1), 21-33. 
Castro Jr, D. F. L. (2013). Estratégias para a hotelaria executiva catarinense: uma análise entre as capacidades mercadológicas, diferenciação de serviços e desempenho. Tese de Doutorado, Programa de Pós-Graduação em Administração, Universidade do Vale do Itajaí, Biguaçu, SC, Brasil..

Eisenhardt, K. M., \& Martin, J. A. (2000). Dynamic capabilities: what are they? Strategic Management Journal, 21, 1105-1121.

Ergün, E., \& Tasgit, Y. E. (2013). Cultures of adhocracy, clan, hierarchy and market and innovation performance: a case of hotels in Turkey. Journal of Travel and Tourism Research, 13 (1/2), 132-142.

Fávero, L. P. L., Belfiore, P. P., Chan, B. L., \& Silva, F. L. (2009). Análise de Dados: Modelagem Multivariada para Tomada de Decisões (1a ed.). Rio de Janeiro: Elsevier.

Hair, J. F. Jr., Anderson, R. E., Tatham, R. L, \& Black, W. C. (2009). Análise multivariada de dados. (6a ed.). Porto Alegre: Bookman.

Haveman, H. A. (1992). Between a rock and a hard place: organizational change and performance under conditions of fundamental environmental transformation. Administrative Science Quartely, 37(1), 48-75.

Helfat, C. E., Finkelstein, S., Mitchell, W., Peteraf, M., Singh, H., Teece, D. \& Winter, S. (2007). Dynamic Capabilities: Understanding Strategic Change in Organizations. London: Blackwell.

Hu, X., Zhou, X., Dai, W., Zhan, Z., \& Liu, X. (2012). Tourism crisis management system based on ecological mechanism. Journal of Software, 7(12), 2808-2815.

Hung, R. Y-Y., Chung, T., \& Lien, B. Y-H. (2007). Organizational process alignment and dynamic capabilities in high-tech industry. Total Quality Management, 18(9), 1023-1034.

Kala, D., \& Bagri, S. C. (2014). Key performance indicators for hospitality industry: a study from the tourist state of Uttarakhandm, Indian. Tourismos: an International Multidisciplinaray Journal of Tourism, 9(1), 187-206.

Kiessling, T., \& Harvey, M. (2004). Global marketing networks and the development of trust: a dynamic capabilities perspective. Journal of Marketing Channels, 11(4), 21-41.

Krzakiewicz, K. (2013). Dynamic capabilities and knowledge management. Management, 2(17), 1-15.

Limberger, P. F., Boaria, F., \& Anjos, S. J. G. (2014). A relação entre a satisfação geral e as variáveis da satisfação na hotelaria em hotéis de excelência. Revista Brasileira de Pesquisa em Turismo, 8(3), 435-455.

Makkonen, H., Pohjola, M., Olkkonen, R., \& Koponen, A. (2014). Dynamic capabilities and firm performance in a financial crisis. Journal of Business Research, 67(2014).

Marquesan, F. F. S., Guzzo, R. F., Zawislak, P. A., \& Tello-Gamarra, J. (2015). A importância dos ativos específicos na diferenciação de firmas do setor hoteleiro. E\&G Economia e Gestão, 15(41), 79-111.

Muthén, L. K., \& Muthén, B. O. (2010). Mplus User's Guide. (6a ed.). Los Angeles: Muthén \& Muthén.

Ng, J. C. Y., \& Tan, H. T. (2015). Going global: do you have the necessary strategic and resources fit? Indian Journal of Management Science (IJMS), 5(1), 96-100.

Nieves, J., Haller, S. (2014). Building dynamic capabilities through knowledge resources. Tourism Management, 40, 224-232.

Pillips, P., \& Moutinho, L. (2014). Critical review of strategic planning research in hospitality and tourism. Annals of Tourism Research, 48, 96-120.

Silveira-Martins, E., Rossetto, C. R., \& Añaña, E. (2014). Ambidestria, exploração ou explotação e seus efeitos no desempenho organizacional de vinícolas brasileiras. Revista em Agronegócios e Meio Ambiente, 7(3), 707732.

Silveira-Martins, E., \& Tavares, P. M. (2014a). Processo de formulação de estratégias: capacidade mercadológica, incerteza ambiental e desempenho. Organizações em contexto, 10(20), 297-322.

Silveira-Martins, E., \& Tavares, P. M. (2014b). Associação entre capacidades gerenciais e desempenho mediadas pela incerteza ambiental: um estudo na Agrox. Revista Cesumar Ciências Humanas e Sociais Aplicadas, 19(2), 347-370.

Silveira-Martins, E., \& Vaz, C. S. (2016). Orientação empreendedora e sua associação com as capacidades dinâmicas: um estudo em agroindústrias gaúchas. Revista em Agronegócios e Meio Ambiente, no prelo.
Silveira-Martins, E., \& Zonatto, V. C. S. (2016). Proposição e validação de escala para mensuração de capacidade turística. Revista Turismo em Análise, prelo.

Tachizawa, T., Pozo, H., \& Vicente, A. J. (2013). O uso de tecnologias da informação em hotéis de pequeno porte: um estudo multicaso. Organizações em Contexto, 9(17), 31-57.

Teece, D., \& Al-Aali, A. Y. (2013). Knowledge, entrepreneurship, and capabilities: revising the theory of the MNE. Universia Business Review, 40, 18-32.

Teece, D., \& Pisano, G. (1994). The dynamic capabilities of firms: an introduction. Industrial and Corporate Change, 3(3), 537-556.

Teece, D., Pisano, G., \& Shuen, A. (1997). Dynamic capabilities and strategic management. Strategic Management Journal, 18(7), 509-533.

Tondolo, V. A. G., \& Bitencourt C. C. (2014). Compreendendo as capacidades dinâmicas a partir de seus antecedentes, processos e resultados. Brazilian Business Review, 11(5), 124-147.

Vaz, C. S., \& Silveira-Martins, E. (2016). Capacidades dinâmicas associadas ao desempenho: um estudo realizado em agroindústrias gaúchas. Revista Espacios, 37(6), 2016.

Walsh, M., Lynch, P., \& Harrington, D. (2011). A capability-based framework for tourism innovativeness. Irish Journal of Management, 31(1), 21-41.

Wang, C. L., \& Ahmed, P. K. (2007). Dynamic capabilities: a review and research agenda. International Journal of Management Reviews, 9(1), 31-51.

Wilden, R., Gudergan, S. P., Nielsen, B. B., \& Lings, I. (2013). Dynamic capabilities and performance: strategy, structure and environment. Long Range Planning, 46(2013), 72-96.

Recebido: 28.02.2016

Reenviado: 11.07.2016

Aceite: 25.07.2016 